Canadian Science Publishing

Biochemistry and Cell Biology

Biochimie et biologie cellulaire

\title{
Lactoferrin and lactoferrin-sophorolipids-assembly can be internalized by dermal fibroblasts and regulate gene expression
}

\begin{tabular}{|r|l|}
\hline Journal: & Biochemistry and Cell Biology \\
\hline Manuscript ID & bcb-2016-0090.R3 \\
\hline Manuscript Type: & Article \\
\hline Date Submitted by the Author: & 07-Nov-2016 \\
\hline Complete List of Authors: & $\begin{array}{l}\text { Jiang, Rulan; University of California at Davis } \\
\text { Suzuki, Yasushi; Saraya Co. Ltd., Biochemical laboratory } \\
\text { Du, Xiaogu; University of California, Davis, Nutrition } \\
\text { Lonnerdal, Bo; University of California at Davis }\end{array}$ \\
\hline Keyword: & $\begin{array}{l}\text { lactoferrin, sophorolipids, dermal fibroblasts, internalization, gene } \\
\text { expression }\end{array}$ \\
\hline &
\end{tabular}


1 Lactoferrin and lactoferrin-sophorolipids-assembly can be internalized by

2 dermal fibroblasts and regulate gene expression

3

4 Rulan Jiang ${ }^{1}$, Yasushi A. Suzuki ${ }^{2}$, Xiaogu $\mathrm{Du}^{1}$, and Bo Lönnerdal ${ }^{1}$

$5{ }^{1}$ Department of Nutrition, University of California, Davis; ${ }^{2}$ Biochemical Laboratory,

6 Saraya Co. Ltd., 24-12 Tamate, Kashiwara, Osaka

7

8

9

10

11

12

13

14

15

16

17 Address correspondence to:

18 Bo Lönnerdal, PhD

19 Department of Nutrition

20 University of California

21 Davis CA 95616, USA

22 Phone: (530) 752-8347

23 Fax: (530) 752-3564

24 E-mail:bllonnerdal@ucdavis.edu 
Abstract: Lactoferrin (Lf) is an iron-binding multifunctional protein, mainly present in external secretions. Lf is known to penetrate skin and may thus exert its multiple functions in skin. Sophorolipids (SLs) are glycolipid biosurfactants, which have been shown to enhance absorption of commercial bovine Lf (CbLf) in model skin via forming an assembly with CbLf. In the present study, uptake and post-internalization localization of bovine Lf (bLf), CbLf, and human Lf (hLf) with or without forming assemblies with SLs in human dermal fibroblasts (HDFn) were determined using ${ }^{125}$-labeled Lfs and confocal microscopy, respectively. Our results show that all three Lfs were internalized by HDFn; although SLs did not significantly affect uptake of Lfs, it changed Lf localization by accumulating Lfs to the perinuclear region. Furthermore, microarrays were used to investigate transcriptional profiling in HDFn in response to CbLf, SLs, or CbLf-SLs-assembly treatments. Transcriptome profiling indicates that CbLf may play roles in protection of skin from oxidative stress, immunomodulatory activities, and enhancement of wound healing. The assembly had similar effects but dramatically modulated transcription of some genes. SLs alone modified signaling pathways related to lipid metabolism, as well as synthesis of sex hormones and vitamins. Thus, CbLf may exert beneficial effects on skin, and these effects may be modulated by SLs.

Key words: lactoferrin, sophorolipids, dermal fibroblasts, internalization, gene expression 


\section{Introduction}

Lactoferrin (Lf) is an iron-binding protein mainly present in external secretions such as breast milk, saliva, tears, bile, and pancreatic fluids (Lönnerdal 2009). It is a

50 multifunctional protein involved in a wide range of activities, such as immunomodulation,

51 and promotion of cell proliferation (Vogel 2012). Effects of Lf on skin comprise

52 promotion of skin wound healing (Takayama and Aoki 2012), improvement of 53 inflammatory lesions of acne vulgaris (Kim et al. 2010; Mueller et al. 2011), up54 regulation of proliferation of dermal fibroblasts and keratinocytes (Engelmayer et al. 55 2008; Tang et al. 2010), and attenuation of UV-induced photo damage (Murata et al.

57 Therefore, it may be feasible to add Lf to water-based cosmetics. In general, Lf exerts 58 its multiple functions via two mechanisms; 1) working as a transcription factor to 59 transactivate gene expression (He and Furmanski 1995), or 2) binding to a specific

65 also function diversely because they have markedly different conformations

66 (Grossmann et al. 1992). For this reason, apo- and holo- forms of several Lfs were 67 investigated in the present study. 
71

72

73

74

75

76

77

78

79

80

81

82

\section{3}

84

85

86

87

88

89

90 91

and a long chain hydroxyl fatty acid with 16 or 18 carbon atoms. SLs can form a vesicle-like structure to promote transport of substances into cells through size reduction and encapsulation (Imura et al. 2014; Zhou et al. 2004). SLs produced by Starmerella bombicola have been shown to enhance CbLf absorption by model skin up to 1.7-fold via forming an assembly with CbLf. Moreover, SLs significantly regulated gene expression upon the CbLf treatment (Ishii et al. 2012). In addition to being biodegradable surfactants, SLs possess skin compatibility, anti-inflammation (Bluth et al. 2006), and antimicrobial activities (Azim et al. 2006; Diaz De Rienzo et al. 2016; Solaiman et al. 2015), as well as good moisturizing and depigmenting properties (Lundov et al. 2009). Therefore, SLs have been applied in various cosmetic formulations (Morya et al. 2013; Van Bogaert et al. 2007; Varvaresou and lakovou 2015).

The objectives of the present study were to examine uptake and postinternalization localization of apo- and holo-Lfs, as well as effects of Lfs and SLs on dermal fibroblasts, and thus to provide preliminary data and a scientific basis for addition of Lf and SLs to cosmetic products. To address the objectives, we examined whether hLf, bLf, and CbLf were internalized by human dermal fibroblasts (HDFn) by using I-125 labeled Lfs and the post-internalization localization of Lfs in HDFn by confocal microscopy. The microarray is a powerful tool to identify genes simultaneously relevant to various treatments (Papp et al. 2012), and we therefore used microarrays to characterize global transcription profiles of CbLf-, SLs-, and CbLf-SLs-assemblytreated human dermal fibroblasts (HDFn). 


\section{Materials and methods}

\section{Preparation of hLf and bLf}

Human-Lf (hLf) and bovine Lf (bLf) were isolated from milk as described previously (Lönnerdal et al. 2011). To examine the purity and concentration of Lf in each eluted fraction, each sample $(10 \mu \mathrm{L})$ was subjected to SDS-PAGE and gels were

97 stained with Coomassie Brilliant Blue R-250 (Sigma, St Louis, MO). Based on the gel results, only Lf isolated from fraction 2 with an elution buffer containing the higher

99 concentration of $\mathrm{NaCl}(1.0 \mathrm{~mol} / \mathrm{L})$ was used for subsequent experiments. CbLf $(\sim 15 \%$ 100

101 Iron-free Lf (Apo-Lf) and iron saturated Lf (holo-Lf) were prepared as described 102 previously (Lopez et al. 2008; van Berkel et al. 1995).

Preparation of SLs and Lf-SLs-assemblies

SLs were produced by Starmerella bombicola NBRC10243. Stock solutions of SLs and Lf-SLs-assemblies were prepared using methods described previously (Ishii et 106 al. 2012).

107 Cell culture

Human dermal fibroblasts (HDFn, Cascade Biologics, Portland, OR) were 109 maintained in a humidified incubator at $37^{\circ} \mathrm{C}$ under an atmosphere of $5 \% \mathrm{CO}_{2}$ in 110 Medium 106 supplemented with Low Serum Growth Supplement (LSGS, Cascade 111 Biologics, Portland, OR). The medium was changed every other day, and cells between 112 passages 5-10 were used in the present study. 


\section{Lf uptake}

All Lfs were labeled with ${ }^{125}$ (GE Healthcare, Waukesha, WI) using the lodogen (Pierce, Rockford,IL) method. HDFn were seeded on 24-well plates $\left(2.5 \times 10^{3} \mathrm{cells} / \mathrm{cm}^{2}\right)$. After cells were cultured for 10 days, D10 HDFn on 24-well plates were incubated in supplement-free medium (SFM) containing ${ }^{125}$ I-labeled Lf samples $(100 \mu \mathrm{g} / \mathrm{mL})$, or LfSLs-assembly $(100 \mu \mathrm{g} / \mathrm{mL} \mathrm{Lf}$ and $10 \mu \mathrm{g} / \mathrm{mL} \mathrm{SLs})$ for $30 \mathrm{~min}$ at $37^{\circ} \mathrm{C}$. Cells were rinsed with ice-cold PBS 3 times and then liquefied with $\mathrm{NaOH}(1 \mathrm{~mol} / \mathrm{L})$. After cells were solubilized, cell-associated radioactivity was quantified in a gamma counter (Packard Minaxi $\mathrm{Y}$; Meriden, CT). Non-specific binding was assessed by incubation with unlabeled Lf (100-fold molar excess) and subtracted from total uptake, resulting in specific uptake.

\section{Indirect fluorescence}

HDFn were seeded and grown on Lab-Tekll chambered slides (Nalge Nunc International, Naperville, IL) overnight. After cells were treated with Lfs $(100 \mu g / m L), ~ L f$ assemblies $(100 \mu \mathrm{g} / \mathrm{mL})$, or SLs $(10 \mu \mathrm{g} / \mathrm{mL})$ in SFM for 30 min at $37^{\circ} \mathrm{C}$, cells were rinsed with PBS, fixed with phosphate-buffered paraformaldehyde $(4 \%, 0.4 \mathrm{~mL} /$ well) for $10 \mathrm{~min}$ at room temperature, and then permeabilized with Triton $\mathrm{X}-100(0.2 \%)$ in PBS for 10 min. Cells were subsequently blocked with blocking buffer ( $5 \%$ heat-inactivated rabbit serum and 1\% BSA in PBS, $0.5 \mathrm{~mL} /$ well) for $20 \mathrm{~min}$. After the blocking buffer was removed, cells were rinsed with PBS. Lf was subsequently probed with rabbit anti-hLf (2 $\mu \mathrm{g} / \mathrm{mL}$, Abcam, Cambridge, MA), or goat anti-bLf $(2 \mu \mathrm{g} / \mathrm{mL}$, Bethyl Laboratories, Montgomery, TX), and then Alexa 488-conjugated-anti-rabbit or Alexa 488-conjugated- 
135 anti-goat $\operatorname{lgG}(1 \mu \mathrm{g} / \mathrm{mL}$; Molecular Probes, Eugene, OR) in blocking buffer for $30 \mathrm{~min}$.

136 The nucleus was stained with TOPO-3 (1:1000 in blocking buffer, Molecular Probe,

137 Eugene, Oregon) for $30 \mathrm{~min}$. After several rinses, coverslips were mounted with

138 ProLong Gold Antifade Reagent (Invitrogen Molecular Probes) and sealed with nail 139 polish. A confocal laser scanning microscope (FV1000, Olympus America, Inc., Melville, $140 \mathrm{NY}$ ) was used to perform immunofluorescence imaging, and image analysis software 141 systems (Olympus America, Inc.) were applied to analyze the images. The density of Lf 142 fluorescence and the ratio of perinuclear to total internalized Lf in 20 cells from four 143 slides for each treatment group were measured by using analytical tools from Image $\mathrm{J}$ 144 (http://imagej.nih.gov/ij/). The perinuclear area was defined as the cytoplasmic area 145 encircling the nucleus and having a radius around $10 \mu \mathrm{m}$.

146 RNA extraction and array hybridization

HDFn (6-well plate, $2.5 \times 10^{3} / \mathrm{cm}^{2}$ ) were grown for $24 \mathrm{~h}$ and then treated with 148 CbLf $(100 \mu \mathrm{g} / \mathrm{mL})$, SLs $(10 \mu \mathrm{g} / \mathrm{mL})$, or CbLf-SLs-assembly for $24 \mathrm{~h}$. RNA was subsequently extracted with Trizol (Invitrogen), and then purified by the RNease kit 150 (Qiagen) according to the manufacturer's instructions. Measurement of RNA yield was 151 performed using a NanoDrop 1000A Spectrophotometer (Thermo Fisher Scientific, 152 Waltham, MA), and RNA quality was evaluated by Bioanalyzer RNA Nano Chips 153 (Agilent Technologies, Inc., Santa Clara, CA) following the manufacturer's procedure. 154 Total RNA samples were amplified and labeled with biotinylated nucleotides using a kit 155 (Ambion, TotalPrep -96 RNA Amplification Kit). Bioanalyzer analysis was then carried 156 out to verify if cRNA was at the expected the $1.2 \mathrm{~kb}$ average size before applying to 
157 beadchips (HumanHT-12 v.4.0, Illumina, San Diego). Beadchips were scanned with the 158 Illumina iScan using standard conditions.

\section{Microarray data analysis}

GenomeStudio (Illumina) was utilized for microarray data analysis and quantile normalization was carried out to normalize the microarray data. Only probe sets with 162 adjusted $p$-value $<0.05$ were selected for subsequent analysis and totally 13716 genes were analyzed. The differentially expressed genes were selected following two criteria:

164 fold-change $>1.5$ for up-regulation, and fold-change $<0.5$ for down-regulation. Samples 165 were clustered with a centroid linkage method on the horizontal axis, and the lengths of 166 the branches were measure by a Euclidean algorithm. Pathway analysis was conducted 167 using IPA (Ingenuity ${ }^{\circledR}$ Systems, www.ingenuity.com) and the Core analysis included in 168 IPA was chosen to interpret data in the context of biological processes, pathways, and 169 networks. A p-value was calculated using the right-tailed Fishers exact test for each 170 canonical pathway and $p$-values less than 0.05 were considered significantly relevant. 171 Heatmapper (http://www.heatmapper.ca/) was used to generate the heatmap.

172 The accession number

173 Microarray results have been deposited in the NCBI Gene Expression Omnibus 174 (http://www.ncbi.nlm.nih.gov/geo/) and are available through the accession number 175 (GSE84144).

\section{Quantitative real-time PCR}


RNA $(1 \mu \mathrm{g})$ was reverse-transcribed to cDNA using a high-capacity cDNA 178 reverse transcription kit (Applied Biosystems, Foster City, CA) following the 179 manufacturer's instructions. Gene-specific primers are listed in table 1. Real-time 180 polymerase chain reaction (RT-PCR) was performed on the cDNA reaction mixture (2 $181 \mu \mathrm{L}$ ) and SYBR Green (Thermo Scientific) using the iCycler real-time PCR system (Bio182 Rad). The cycling parameters were $95^{\circ} \mathrm{C} 15 \mathrm{~min}$ and 40 cycles including $95^{\circ} \mathrm{C}$ for 15 $183 \mathrm{sec}, 60^{\circ} \mathrm{C}$ for $30 \mathrm{sec}$, and $72^{\circ} \mathrm{C}$ for $30 \mathrm{sec}$. Linearity of the dissociation curve was 184 analyzed using the iCycler software and the mean cycle time of the linear part of the 185 curve was designated as Ct. Each sample was analyzed in sextuplicate and normalized 186 to GAPDH using the following equation: fold change $=2\left({ }^{\mathrm{Ct} \mathrm{Gene}-\mathrm{Ct} \mathrm{GAPDH}}\right)$. Values are 187 shown as mean fold change \pm standard deviation, relative to the non-treated control (set 188 to 1$)$.

\section{Statistical analysis}

Data represent means \pm standard deviations from at least 3 independent experiments. Comparisons between the treatment and control were conducted using one-way ANOVA (Prism Graph Pad, Berkeley, CA). P $<0.05$ was considered to be 193 statistically significant.

\section{Results}

\section{Apo- and holo-Lf are internalized by HDFn}

To determine if bLf and CbLf were internalized by HDFn, they were labeled with

${ }^{125}$ I by using the lodogen method. Based on our pilot study, the Lf receptor (LfR), 198 originally isolated from the human small intestine (Suzuki et al. 2001), and LRP1, a 
199 functional LfR in fibroblasts (Takayama et al. 2003), displayed peak expression at day 20010 (data not shown). D10 HDFn were therefore used for uptake experiments. As shown 201 in Fig. 1, CbLf and bLf were internalized by HDFn, and no differences were found in 202 uptake between HDFn incubated with Lfs and Lf-SLs-assemblies. As a comparison, we 203 included hLf and the results were similar to those for the two bovine Lfs. All apo-forms 204 of Lf showed significantly higher uptake than holo-forms of Lf, suggesting that 205 206 highest uptake among all Lfs. Indirect fluorescence detection was next conducted to examine if SLs influence intracellular localization of Lfs after internalization. The results from confocal microscopy confirmed that all Lfs were internalized by HDFn (Fig. 2). The post-internalization

210 localization of all Lfs was altered by SLs as significant amounts of hLf, bLf, and CbLf 211 were localized in the perinuclear area upon treatments with Lf-SLs-assemblies. 212 Localization of Lfs was not affected by iron saturation for any of the Lfs. Although the 213 major part of Lfs was localized in the cytoplasm, co-localization of Lf and the nucleus 214 was observed for all treatments, suggesting that all the Lfs are capable to enter the 215 nucleus of HDFn and thereby exert multiple functions.

\section{Microarray analysis of HDFn treated with CbLf, SLs, and CbLf-SLs-assembly}

Since any practical application of Lf will be dependent upon commercially available bLf, effects of CbLf, SLs, or CbLf-SLs-assembly on HDFn were investigated by microarray analysis. According to the cluster analysis (Fig. 3) and heatmap (Fig. 4), 
221 CbLf-SLs-assembly. The cluster analysis of the entire genome indicates that the three

222 biological replicates were consistent, and that CbLf and CbLf-SLs-assembly displayed 223 similar effects. As shown in Fig. 3, CbLf- and CbLf-SLs-assembly-treated samples 224 formed distinct clusters, separately from the control and SLs-treated samples, indicating 225 that CbLf and CbLf-SLs-assembly significantly altered transcriptional profiles of HDFn, 226 and that SLs-treated HDFn exhibited the weakest transcriptional response. Consistently, 227 the heatmap generated by markedly modified genes also showed that CbLf and CbLf228 SLs-assembly significantly changed gene transcription of HDFn, and similar effects on 229 HDFn were found in presence of CbLf or CbLf-SLs-assembly (Fig.4). significantly regulated gene transcription in HDFn. Total genes modulated by CbLf, SL, 232 and CbLf-SLs-assembly were 1520, 100, and 1472, respectively. 1263 genes, about $23386 \%$ of genes changed by CbLf were modified by both CbLf and CbLf-SLs-assembly, 234 indicating that SLs forming an assembly with CbLf only slightly change effects of CbLf 235 on transcription profiling. Although SLs alone significantly stimulated transcription of 236 some genes, the effects were much weaker than those initiated by CbLf and CbLf-SLs237 assembly.

\section{Validation of microarray results by Real-time PCR}

To verify results from the microarray analysis, six genes (FABP3, COL7A1, CXCL6, PLIN2, CPA4, and EDN1) were selected for real-time PCR analysis in terms of expression intensity and various patterns of gene expression regulated by CbLf, SLs, or

242 CbLf-SLs-assembly (Fig. 6). For example, the FABP3 gene was selected because this 
243 gene was significantly modified by all three treatments, and the EDN1 gene was chosen

244 because only SLs had a significant effect on transcription of this gene. The results from

245 these six genes were consistent with results from the microarrays and the $R^{2}$ of 246 association analysis was higher than 0.95 (Fig. 6G), indicating that the two data sets

247 were strongly correlated. Thus, results from the microarray assays were validated.

\section{Effects of CbLf and CbLf-SLs-assembly on proliferation of HDFn}

CbLf and CbLf-SLs-assembly significantly stimulated transcription of genes 250 involved in cellular proliferation. As shown in Supplemental table 1, these genes 251 comprised kinases, transcription factors, cell cycle regulators, growth factors, and 252 components of the extracellular matrix. CbLf and the assembly showed similar effects, 253 except for few genes on which CbLf had stronger effects than the assembly. Since SLs 254 did not significantly modulate transcription of any of these genes listed in the Table, only 255 results for CbLf and CbLf-SLs-assembly are shown.

According to pathway analysis $(p<0.05)$, a number of signaling pathways induced

257 by CbLf and the assembly are involved in regulating transcription of proliferation related 258 genes (Supplemental table 3). PI3K/AKT, PDGF, and IGF-1 pathways were three major 259 signaling pathways by which CbLf and CbLf-SLs-assembly increased proliferation of 260 HDFn. In addition, CbLf-SLs-assembly activated VEGF signaling to exert its proliferative 261 effects on HDFn.

262 Effects of CbLf and CbLf-SLs-assembly on transcription of genes involved in 263 migration of HDFn 

In agreement with enhancing effects of Lfs on migration of dermal fibroblasts

265 (Engelmayer et al. 2008; Francis et al. 2011), both CbLf and CbLf-SLs-assembly 266 significantly promoted transcription of genes mediating cell migration (Supplemental 267 table 1), including growth factors, extracellular matrix and related enzymes, suggesting 268 that CbLf and CbLf-SLs-assembly may play a role in enhancement of cell migration.

CbLf and CbLf-SLs-assembly up-regulated expression of genes involved in generation of the extracellular matrix

Our results show that CbLf and CbLf-SLs-assembly markedly enhanced 272 transcription of components of the extracellular matrix (ECM) (Supplemental Table 1), 273 which is consistent with previous reports showing that CbLf promoted secretion of 274 hyaluronan in human normal dermal fibroblasts (Saito et al. 2011; Takayama et al. 275 2003). Compared with effects regulated by CbLf, the assembly had stronger or weaker 276 effects on transcription of some genes, such as TIMP, PLOD2, and VCAN. Expression 277 of collagen I, the most abundant collagen in skin, was significantly up-regulated by 278 CbLf. Besides proteins of the ECM, CbLf and CbLf-SLs-assembly also enhanced 279 transcription of enzymes required for construction of ECM, and increased transcription 280 of inhibitors for matrix metalloproteinases (MMPs), which degrade collagens. Put 281 together, CbLf and CbLf-SLs-assembly up-regulated ECM synthesis via promotion of 282 synthesis of ECM components and their required enzymes, as well as up-regulation of 283 MMP inhibitors.

284 CbLf and CbLf-SLs-assembly promoted transcription of genes involved in 285 resistance against oxidative stress 
CbLf and CbLf-SLs-assembly significantly increased the potential for oxidative 287 stress resistance in dermal fibroblasts via stimulating transcription of genes 288 (Supplementary table 1) involved in removal of reactive oxygen species (ROS), 289 particularly superoxide dismutase (SOD). Two signaling pathways modified were found 290 to be involved in antioxidant reactions, namely NRF2 oxidative stress response, and the 291 antioxidant action of vitamin C (Supplementary table 3).

292 Effects of CbLf and CbLf-SLs-assembly on expression of genes involved in 293 Immunomodulatory activities

CbLf and CbLf-SLs-assembly considerably stimulated transcription of genes 295 related to immunomodulatory effects. As shown in Supplementary table 1, expression of 296 genes for cytokines, chemokines, and kinases were dramatically increased. The 297 immunomodulatory effects were mediated by several signaling pathways 298 (Supplementary table 3). Dominant signaling pathways including IL-6, iNOS, Tweak, IL299 8, and IL-17A signaling mediated effects triggered by both CbLf and CbLf-SLs300 assembly.

\section{Effects on lipid metabolism and other effects of SLs on HDFn}

CbLf, CbLf-SLs-assembly, and SLs promoted cholesterol biosynthesis of HDFn 303 (Supplementary tables 2\&4). Although CbLf, assembly, and SLs activated similar 304 signaling pathways to enhance cholesterol biosynthesis, the treatment with SLs alone 305 exhibited the highest efficacy. In addition to the effect on lipid metabolism, SLs 306 enhanced biosynthesis of estrogen, androgen, and vitamin A \&D (Supplementary table 307 $5)$. 
308

\section{Pathway analysis summary}

CbLf and CbLf-SLs-assembly significantly regulated gene transcription profiling of HDFn. A large number of genes involved in diverse biological processes are upregulated in the transcriptional response. Based on the pathway analysis, CbLf and CbLf-SLs-assembly play critical roles in cellular functions (Supplementary table 6). The major functions regulated by CbLf and CbLf-SLs-assembly consisted of positive regulation of cellular assembly and organization, as well as cell cycle progression. The main effects of SLs on dermal fibroblasts comprise regulation of lipid metabolism, vitamin and mineral metabolism, and molecular transport (Supplementary table 7).

\section{Discussion}

Lfs can be internalized by dermal fibroblasts, and SLs may influence effects triggered by CbLf via changing the intracellular localization of internalized Lfs. SLs have been shown to increase CbLf absorption by model skin (Ishii et al. 2012), probably via facilitating transport of CbLf through multiple layers of the epidermis. However, SLs did not affect the rate of CbLf internalization by HDFn as evident by internalization of ${ }^{125}$ labeled Lfs. Instead, SLs changed the intracellular localization of CbLf and may thus affect its physiological functions. Compared with holo-forms of Lfs, all apo-forms of Lfs displayed higher uptake, suggesting that conformation differences play a role in Lf internalization by HDFn and may thereby modify effects of Lfs on dermal fibroblasts. Apo-Lf has less compact conformation than holo-Lf (Baker and Baker 2004), so the LfR binding site in apo-Lf may have higher chances to access the LfR on the membrane of HDFn, leading to higher uptake of apo-Lf by HDFn. 
CbLf and CbLf-SLs-assembly significantly up-regulated transcription of genes

331 involved in cellular proliferation. Major proliferation-related signaling pathways activated

332 by CbLf and CbLf-SLs-assembly were PI3K/AKT, platelet-derived growth factor (PDGF),

333 and insulin-like growth factor (IGF-1) signaling pathways. Additionally, the assembly

334 activated VEGF (vascular endothelial growth factor) signaling. Lf has been reported to

335 stimulate proliferation of dermal fibroblasts (Ishii et al. 2012), and induced proliferation

336 of keratinocytes via activing the ERK signaling pathway (Tang et al. 2010). Fibroblasts

337 are the predominant cells in the dermis, and they play essential roles in maintaining skin

338 homeostasis and forming a heterogeneous cell population which produces ECM

339 components including collagens, elastin, and glycoproteins (Martin 1997). A complex

340 ECM network organized by dermal fibroblasts provides water retention and remarkable

341 biomechanical properties to skin (Vedrenne et al. 2012). Renewal of the epidermis is

342 much slower in elderly due to the unique extension of cell cycles (Kohl et al. 2011), and

343 aging fibroblasts display a slower rate of proliferation than fetal fibroblasts

344 (Khorramizadeh et al. 1999), suggesting that chronological aging at least partly results

345 from decreased replicative capacity of dermal fibroblasts. Thus, promoted proliferation

346 of dermal fibroblasts may result in improvement of skin health through increasing

347 generation of ECM components and stimulating renewal of the epidermis.

CbLf and CbLf-SLs-assembly may be involved in facilitating migration of dermal

349 fibroblasts (Supplementary table 1) via stimulating expression of ECM and related

350 transcription factors, as well as growth factors. The migration of human skin cells is very

351 complex, and it is crucial for skin wound healing and remodeling. Platelet derived

352 growth factor (PDGF) is well characterized for its functions on growth and motility of 
353 dermal fibroblasts, and it is believed to participate in wound healing processes (Li et al.

354 2004). CbLf and CbLf-SLs-assembly dramatically stimulated transcription of both PDGF 355 and PDGF receptor (PDGFRB), suggesting that they may be associated with 356 enhancement of cell migration via increasing PDGF transcription. In addition to PDGF, 357 CbLf and the assembly also enhanced transcription of FGF, another important growth 358 factor related to cell motility ( $\mathrm{Li}$ et al. 2004). Two recombinant hLfs have been shown to 359 promote wound repair via stimulation of cell proliferation and migration of dermal 360 fibroblasts, as well as synthesis of ECM components (Engelmayer et al. 2008; 361 Takayama and Aoki 2012; Tang et al. 2010). Cell migration and proliferation are two 362 essential steps for re-epithelialization in skin wound healing and remodeling (Clark et al. 363 1996). CbLf and CbLf-SLs-assembly significantly enhanced transcription of genes 364 tightly related to cell migration and proliferation in dermal fibroblasts, implying that Lf 365 may play important roles in wound repair.

CbLf and CbLf-SLs-assembly remarkably promoted transcription of genes 367 involved in synthesis of extracellular matrix via enhancing transcription of ECM 368 components, promoting lysyl hydroxylation via stimulating transcription of related 369 enzymes, and inhibiting collagen degradation by increasing transcription of 370 metallopeptidase inhibitor 3 (Supplementary table 1). CbLf has been documented to 371 increase secretion of hyaluronan by increasing transcription of hyaluronan synthase 2 372 (HAS2), and elevate COL1A1 transcriptionin normal human dermal fibroblasts (Saito et 373 al. 2011). Consistently, transcription of HAS2 and collagen I was considerably up374 regulated by CbLf and CbLf-SLs-assembly. In particular, the effect of the assembly on 375 HAS2, a major hyaluronan synthase in dermis, was more potent than that of CbLf. 
376 Collagens I and III are the most abundant collagens present in skin, and lysyl

377 hydroxylation is needed for cross-link formation to stabilize collagen networks in the 378 extracellular space (Krieg and Aumailley 2011), whereas hyaluronic acid fills the 379 connective tissue and is responsible for its strength (Delalle-Lozica 2010). Photo-aged 380 skin contains an abundance of degraded and disorganized collagen fibrils and has a 381 reduced production of type I and type III procollagen (Fisher et al. 2002; Talwar et al. 382 1995). Increased collagen synthesis or protection from collagen breakdown are 383 potential mechanisms for anti-aging effects (Chiu and Kimball 2003).

CbLf and CbLf-SLs-assembly may protect skin from oxidative stress via 385 stimulating expression of related genes and activating anti-oxidative signaling pathways. Covering the entire outer surface of the body, human skin is the largest organ and is constantly exposed to sunlight stress, including ultraviolet (UV) light irradiation. Reactive oxygen species (ROS) damage cellular DNA, lipids, and proteins (Berlett and Stadtman 1997). There are two types of skin aging; one is chronological aging, which is due to the passage of time, and another is premature aging or photo-aging owing to environmental aggressors (Iddamalgoda et al. 2008). ROS generated from oxidative cell metabolism and UV-exposure cause oxidative damage to cellular components, such as cell walls, lipid membranes, mitochondria, and DNA. ROS facilitates both photo-aging and 394 chronological aging (Fisher et al. 2002). Based on the free radical theory, excess ROS generated from oxidative metabolism result in cellular damage, which is a major cause 396 of aging (Hensley and Floyd 2002). CbLf and the assembly significantly activated the 397 NRF2-mediated oxidative stress response and antioxidant action of vitamin C to exert 398 their anti-oxidant effects. Nrf2 is a transcription factor known to initiate a cellular 
399 response to defend cells against the deleterious effects of environmental toxicants 400 (Kensler et al. 2007). Vitamin C is an electron donor, and it eliminates most ROS 401 (Padayatty et al. 2003). CbLf and CbLf-SLs-assembly may remove ROS through 402 activating these two signaling pathways and thus diminish both photo-aging and 403 chronological aging to some extent. In agreement with our results, an in vivo study 404 conducted in hairless mice found that oral supplementation with Lf prevented UV405 induced skin damage (Murata et al. 2014). Consequently, CbLf and CbLf-SLs-assembly 406 treatments may lead to healthy and younger looking skin.

CbLf and CbLf-SLs-assembly may play roles in immunomodulatory activities by 408 enhancing transcription of chemokines, cytokines, and related enzymes. Skin is an effective barrier between the organism and the environment to prevent invasion of 410 pathogens and to protect against toxins (Proksch et al. 2008). CbLf and CbLf-SLs411 assembly may activate IL-6, iNOS, Tweak, IL17A, and IL-8 signaling pathways to 412 prepare or protect dermal fibroblasts against microbial invasion (Supplementary table 3), 413 which may be beneficial for facilitating the wound healing process by reducing the risk 414 of infection. The antimicrobial activities and immunomodulatory effects of Lf have been 415 extensively documented (Siqueiros-Cendon et al. 2014; Vogel 2012; Wakabayashi et al. 416 2014). Microbiological contamination happens easily in cosmetics with high water 417 content, and it causes potential harmful effects to the consumers (Lundov et al. 2009). 418 Addition of Lf to cosmetics may not only prevent microbiological contamination of the 419 product, but also protect the skin from insults from bacteria and viruses. CbLf, CbLf-SLs-assembly, and SLs may all upregulate cholesterol biosynthesis of HDFn. The enhanced cholesterol synthesis may be associated with up-regulation of 
422 hyaluronan synthesis as indicated in Supplementary table 1 , since activities of 423 hyaluronan synthase rely on lipid (Ontong et al. 2014). Moreover, cholesterol is an 424 important ingredient of cell membrane, and it is essential to maintain permeability, 425 fluidity, and protein functions of cell membrane (Espenshade and Hughes 2007). Thus, 426 upregulation of cholesterol may be beneficial for the skin. Additionally, SLs were 427 reported to reduce subcutaneous fat accumulation by increasing leptin synthesis in 428 adipocytes (Lourith and Kanlayavattanakul 2009). Therefore, further studies using 429 animal models are needed to clarify effects of SLs on lipid metabolism.

In addition to the effects on cholesterol biosynthesis, SLs alone were involved in 431 synthesis of sex hormones and vitamins (Fig. 10.). Human skin is both a hormone target 432 and an endocrine gland (Slominski et al. 2013). Estrogens shows a serial positive 433 effects on human skin, such as protection against skin aging, and acceleration of wound 434 healing (Thornton 2005). Moreover, SLs may stimulate synthesis of vitamin A and D, 435 which also have favorable effects on skin. For examples, vitamin A and vitamin D both 436 mediate proliferation and differentiation of skin epithelial cells (Bikle 2015; Randolph 437 and Simon 1997). There are no reported effects of SLs on the synthesis of sex 438 hormones and vitamins, and these effects need to be further validated.

Taken together, Lf is absorbed by dermal fibroblasts and may exert benefical effects on skin health; SLs did not significantly influence internalization and bioactivities 441 of Lf on dermal fibroblasts. Anti-oxidation effects, immunomodulatory activities, and 442 enhancement of wound healing are major possible effects of Lf on dermal fibroblasts 443 (Fig. 7). Lf may exert beneficial roles in skin health by stimulation of proliferation, 444 promotion of life span of dermal fibroblasts, enhancement of synthesis of ECM 
445 components, and increase in oxidative stress resistance. Moreover, Lf may stimulate 446 wound repair as Lf up-regulated transcription of genes involved in promotion of 447 proliferation, and migration of dermal fibroblasts, as well as enhancement of production 448 of ECM components. Topical application of Lf to skin should be further investigated by 449 evaluating changes at the protein level and also in vivo experiments, i.e. animal and 450 human studies.

451

452

453

454

455

456

457

458

459

460

461

462

463

464

465

466

467 


\section{Acknowledgment}

469 We gratefully acknowledge the assistance of Siranoosh Ashtari at the Expression

470 Facility at University of California, Davis with microarray assays.

471

472

473

474

475

476

477

478

479

480

481

482

483

484

485

https://mc06.manuscriptcentral.com/bcb-pubs 


\section{References}

487 Azim, A., Shah, V., Doncel, G.F., Peterson, N., Gao, W., and Gross, R. 2006. Amino 488 acid conjugated sophorolipids: A new family of biologically active functionalized 489 glycolipids. Bioconjug Chem 17(6): 1523-1529. doi: 10.1021/bc060094n.

490 Baker, H.M., and Baker, E.N. 2004. Lactoferrin and iron: structural and dynamic aspects 491 of binding and release. Biometals 17(3): 209-216.

492 Berlett, B.S., and Stadtman, E.R. 1997. Protein oxidation in aging, disease, and 493 oxidative stress. J Biol Chem 272(33): 20313-20316.

494 Bikle, D.D. 2015. Vitamin D receptor, a tumor suppressor in skin. Can J Physiol 495 Pharmacol 93(5): 349-354. doi: 10.1139/cjpp-2014-0367.

496 Bluth, M.H., Kandil, E., Mueller, C.M., Shah, V., Lin, Y.Y., Zhang, H., Dresner, L., 497 Lempert, L., Nowakowski, M., Gross, R., Schulze, R., and Zenilman, M.E. 2006. 498 Sophorolipids block lethal effects of septic shock in rats in a cecal ligation and puncture 499 model of experimental sepsis. Crit Care Med 34(1): 188-195.

500 Chiu, A., and Kimball, A.B. 2003. Topical vitamins, minerals and botanical ingredients 501 as modulators of environmental and chronological skin damage. $\mathrm{Br} \mathrm{J}$ Dermatol 149(4): 502 681-691.

503 Clark, R.A., Tonnesen, M.G., Gailit, J., and Cheresh, D.A. 1996. Transient functional 504 expression of alphaVbeta 3 on vascular cells during wound repair. Am J Pathol 148(5): $505 \quad 1407-1421$.

506 Delalle-Lozica, N. 2010. Local therapy as basic anti-aging prevention. Acta Clin Croat 507 49(4): 529-536. 
508 Diaz De Rienzo, M.A., Stevenson, P., Marchant, R., and Banat, I.M. 2016. Antibacterial 509 properties of biosurfactants against selected Gram-positive and -negative bacteria. 510 FEMS Microbiol Lett 363(2). doi: 10.1093/femsle/fnv224.

511 Engelmayer, J., Blezinger, P., and Varadhachary, A. 2008. Talactoferrin stimulates 512 wound healing with modulation of inflammation. J Surg Res 149(2): 278-286. doi: $513 \quad 10.1016 / j . j s s .2007 .12 .754$.

514 Espenshade, P.J., and Hughes, A.L. 2007. Regulation of sterol synthesis in eukaryotes. 515 Annu Rev Genet 41: 401-427. doi: 10.1146/annurev.genet.41.110306.130315.

516 Fisher, G.J., Kang, S., Varani, J., Bata-Csorgo, Z., Wan, Y., Datta, S., and Voorhees, 517 J.J. 2002. Mechanisms of photoaging and chronological skin aging. Arch Dermatol 518 138(11): 1462-1470.

519 Francis, N., Wong, S.H., Hampson, P., Wang, K., Young, S.P., Deigner, H.P., Salmon, 520 M., Scheel-Toellner, D., and Lord, J.M. 2011. Lactoferrin inhibits neutrophil apoptosis 521 via blockade of proximal apoptotic signaling events. Biochim Biophys Acta 1813(10): 522 1822-1826. doi: 10.1016/j.bbamcr.2011.07.004.

523 Grossmann, J.G., Neu, M., Pantos, E., Schwab, F.J., Evans, R.W., Townes-Andrews, 524 E., Lindley, P.F., Appel, H., Thies, W.G., and Hasnain, S.S. 1992. X-ray solution 525 scattering reveals conformational changes upon iron uptake in lactoferrin, serum and 526 ovo-transferrins. J Mol Biol 225(3): 811-819.

$527 \mathrm{He}, \mathrm{J}$. , and Furmanski, P. 1995. Sequence specificity and transcriptional activation in 528 the binding of lactoferrin to DNA. Nature 373(6516): 721-724. doi: 10.1038/373721a0. 
529 Hensley, K., and Floyd, R.A. 2002. Reactive oxygen species and protein oxidation in 530 aging: a look back, a look ahead. Arch Biochem Biophys 397(2): 377-383. doi: 531 10.1006/abbi.2001.2630.

532 Iddamalgoda, A., Le, Q.T., Ito, K., Tanaka, K., Kojima, H., and Kido, H. 2008. Mast cell 533 tryptase and photoaging: possible involvement in the degradation of extra cellular matrix 534 and basement membrane proteins. Arch Dermatol Res 300 Suppl 1: S69-76. doi: 535 10.1007/s00403-007-0806-1.

536 Imura, T., Morita, T., Fukuoka, T., Ryu, M., Igarashi, K., Hirata, Y., and Kitamoto, D. 537 2014. Spontaneous vesicle formation from sodium salt of acidic sophorolipid and its 538 application as a skin penetration enhancer. J Oleo Sci 63(2): 141-147.

539 Ishii, N., Kobayashi, T., Matsumiya, K., Ryu, M., Hirata, Y., Matsumura, Y., and Suzuki, 540 Y.A. 2012. Transdermal administration of lactoferrin with sophorolipid. Biochem Cell Biol 541 90(3): 504-512. doi: 10.1139/011-065.

542 Jiang, R., and Lönnerdal, B. 2014. Transcriptomic profiling of intestinal epithelial cells in 543 response to human, bovine and commercial bovine lactoferrins. Biometals 27(5): 831544 841. doi: 10.1007/s10534-014-9746-3.

545 Kensler, T.W., Wakabayashi, N., and Biswal, S. 2007. Cell survival responses to 546 environmental stresses via the Keap1-Nrf2-ARE pathway. Annu Rev Pharmacol Toxicol 547 47: 89-116. doi: 10.1146/annurev.pharmtox.46.120604.141046.

548 Khorramizadeh, M.R., Tredget, E.E., Telasky, C., Shen, Q., and Ghahary, A. 1999. 549 Aging differentially modulates the expression of collagen and collagenase in dermal 550 fibroblasts. Mol Cell Biochem 194(1-2): 99-108. 
551 Kim, J., Ko, Y., Park, Y.K., Kim, N.I., Ha, W.K., and Cho, Y. 2010. Dietary effect of

552 lactoferrin-enriched fermented milk on skin surface lipid and clinical improvement of

553 acne vulgaris. Nutrition 26(9): 902-909. doi: 10.1016/j.nut.2010.05.011.

554 Kohl, E., Steinbauer, J., Landthaler, M., and Szeimies, R.M. 2011. Skin ageing. J Eur 555 Acad Dermatol Venereol 25(8): 873-884. doi: 10.1111/j.1468-3083.2010.03963.x.

556 Krieg, T., and Aumailley, M. 2011. The extracellular matrix of the dermis: flexible 557 structures with dynamic functions. Exp Dermatol 20(8): 689-695. doi: 10.1111/j.1600$558 \quad$ 0625.2011.01313.x.

559 Li, W., Fan, J., Chen, M., and Woodley, D.T. 2004. Mechanisms of human skin cell 560 motility. Histol Histopathol 19(4): 1311-1324.

561 Lönnerdal, B. 2009. Nutritional roles of lactoferrin. Curr Opin Clin Nutr Metab Care 562 12(3): 293-297. doi: 10.1097/MCO.0b013e328328d13e.

563 Lönnerdal, B., Jiang, R., and Du, X. 2011. Bovine lactoferrin can be taken up by the 564 human intestinal lactoferrin receptor and exert bioactivities. J Pediatr Gastroenterol Nutr 565 53(6): 606-614. doi: 10.1097/MPG.0b013e318230a419.

566 Lopez, V., Kelleher, S.L., and Lönnerdal, B. 2008. Lactoferrin receptor mediates apo567 but not holo-lactoferrin internalization via clathrin-mediated endocytosis in trophoblasts.

568 Biochem J. doi: BJ20070393 [pii]

569 10.1042/BJ20070393.

570 Lourith, N., and Kanlayavattanakul, M. 2009. Natural surfactants used in cosmetics: 571 glycolipids. Int J Cosmet Sci 31(4): 255-261. doi: 10.1111/j.1468-2494.2009.00493.x. 
572 Lundov, M.D., Moesby, L., Zachariae, C., and Johansen, J.D. 2009. Contamination

573 versus preservation of cosmetics: a review on legislation, usage, infections, and contact

574 allergy. Contact Dermatitis 60(2): 70-78. doi: 10.1111/j.1600-0536.2008.01501.x.

575 Martin, P. 1997. Wound healing--aiming for perfect skin regeneration. Science 576 276(5309): 75-81.

577 Morya, V.K., Ahn, C., Jeon, S., and Kim, E.K. 2013. Medicinal and cosmetic potentials 578 of sophorolipids. Mini Rev Med Chem 13(12): 1761-1768.

579 Mueller, E.A., Trapp, S., Frentzel, A., Kirch, W., and Brantl, V. 2011. Efficacy and 580 tolerability of oral lactoferrin supplementation in mild to moderate acne vulgaris: an 581 exploratory study. Curr Med Res Opin 27(4): 793-797. doi: $582 \quad 10.1185 / 03007995.2011 .557720$.

583 Murata, M., Satoh, T., Wakabayashi, H., Yamauchi, K., Abe, F., and Nomura, Y. 2014. 584 Oral administration of bovine lactoferrin attenuates ultraviolet B-induced skin 585 photodamage in hairless mice. J Dairy Sci 97(2): 651-658. doi: 10.3168/jds.2013-7153.

586 Ontong, P., Hatada, Y., Taniguchi, S., Kakizaki, I., and Itano, N. 2014. Effect of a 587 cholesterol-rich lipid environment on the enzymatic activity of reconstituted hyaluronan 588 synthase. Biochem Biophys Res Commun 443(2): 666-671. doi: $589 \quad$ 10.1016/j.bbrc.2013.12.028.

590 Padayatty, S.J., Katz, A., Wang, Y., Eck, P., Kwon, O., Lee, J.H., Chen, S., Corpe, C., 591 Dutta, A., Dutta, S.K., and Levine, M. 2003. Vitamin C as an antioxidant: evaluation of 592 its role in disease prevention. J Am Coll Nutr 22(1): 18-35. 
593 Papp, K., Szittner, Z., and Prechl, J. 2012. Life on a microarray: assessing live cell 594 functions in a microarray format. Cell Mol Life Sci 69(16): 2717-2725. doi: 595 10.1007/s00018-012-0947-z.

596 Proksch, E., Brandner, J.M., and Jensen, J.M. 2008. The skin: an indispensable barrier. 597 Exp Dermatol 17(12): 1063-1072.

598 Randolph, R.K., and Simon, M. 1997. Metabolism of all-trans-retinoic acid by cultured 599 human epidermal keratinocytes. J Lipid Res 38(7): 1374-1383.

600 Saito, S., Takayama, Y., Mizumachi, K., and Suzuki, C. 2011. Lactoferrin promotes 601 hyaluronan synthesis in human dermal fibroblasts. Biotechnol Lett 33(1): 33-39. doi: 602 10.1007/s10529-010-0389-3.

603 Siqueiros-Cendon, T., Arevalo-Gallegos, S., Iglesias-Figueroa, B.F., Garcia-Montoya, 604 I.A., Salazar-Martinez, J., and Rascon-Cruz, Q. 2014. Immunomodulatory effects of 605 lactoferrin. Acta Pharmacol Sin 35(5): 557-566. doi: 10.1038/aps.2013.200.

606 Slominski, A., Zbytek, B., Nikolakis, G., Manna, P.R., Skobowiat, C., Zmijewski, M., Li, 607 W., Janjetovic, Z., Postlethwaite, A., Zouboulis, C.C., and Tuckey, R.C. 2013. 608 Steroidogenesis in the skin: implications for local immune functions. J Steroid Biochem 609 Mol Biol 137: 107-123. doi: 10.1016/j.jsbmb.2013.02.006.

610 Solaiman, D.K., Ashby, R.D., and Crocker, N.V. 2015. High-titer production and strong 611 antimicrobial activity of sophorolipids from Rhodotorula bogoriensis. Biotechnol Prog 612 31(4): 867-874. doi: 10.1002/btpr.2101.

613 Suzuki, Y.A., Shin, K., and Lonnerdal, B. 2001. Molecular cloning and functional 614 expression of a human intestinal lactoferrin receptor. Biochemistry 40(51): 1577161515779. 
616 Takayama, Y., and Aoki, R. 2012. Roles of lactoferrin on skin wound healing. Biochem 617 Cell Biol 90(3): 497-503. doi: 10.1139/o11-054.

618 Takayama, Y., Takahashi, H., Mizumachi, K., and Takezawa, T. 2003. Low density 619 lipoprotein receptor-related protein (LRP) is required for lactoferrin-enhanced collagen 620 gel contractile activity of human fibroblasts. J Biol Chem 278(24): 22112-22118. doi: 621 10.1074/jbc.M300894200.

622 Talwar, H.S., Griffiths, C.E., Fisher, G.J., Hamilton, T.A., and Voorhees, J.J. 1995. 623 Reduced type I and type III procollagens in photodamaged adult human skin. J Invest 624 Dermatol 105(2): 285-290.

625 Tang, L., Wu, J.J., Ma, Q., Cui, T., Andreopoulos, F.M., Gil, J., Valdes, J., Davis, S.C., 626 and Li, J. 2010. Human lactoferrin stimulates skin keratinocyte function and wound re627 epithelialization. Br J Dermatol 163(1): 38-47. doi: 10.1111/j.1365-2133.2010.09748.x.

628 Thornton, M.J. 2005. Oestrogen functions in skin and skin appendages. Expert Opin 629 Ther Targets 9(3): 617-629. doi: 10.1517/14728222.9.3.617.

630 van Berkel, P.H., Geerts, M.E., van Veen, H.A., Kooiman, P.M., Pieper, F.R., de Boer, 631 H.A., and Nuijens, J.H. 1995. Glycosylated and unglycosylated human lactoferrins both 632 bind iron and show identical affinities towards human lysozyme and bacterial 633 lipopolysaccharide, but differ in their susceptibilities towards tryptic proteolysis. Biochem 634 J 312 (Pt 1): 107-114.

635 Van Bogaert, I.N., Saerens, K., De Muynck, C., Develter, D., Soetaert, W., and 636 Vandamme, E.J. 2007. Microbial production and application of sophorolipids. Appl 637 Microbiol Biotechnol 76(1): 23-34. doi: 10.1007/s00253-007-0988-7. 
638 Varvaresou, A., and lakovou, K. 2015. Biosurfactants in cosmetics and 639 biopharmaceuticals. Lett Appl Microbiol 61(3): 214-223. doi: 10.1111/lam.12440.

640 Vedrenne, N., Coulomb, B., Danigo, A., Bonte, F., and Desmouliere, A. 2012. The 641 complex dialogue between (myo)fibroblasts and the extracellular matrix during skin 642 repair processes and ageing. Pathol Biol (Paris) 60(1): 20-27. doi: 643 10.1016/j.patbio.2011.10.002.

644 Vogel, H.J. 2012. Lactoferrin, a bird's eye view. Biochem Cell Biol 90(3): 233-244. doi: $645 \quad 10.1139 / 02012-016$.

646 Wakabayashi, H., Oda, H., Yamauchi, K., and Abe, F. 2014. Lactoferrin for prevention 647 of common viral infections. J Infect Chemother 20(11): 666-671. doi: $648 \quad$ 10.1016/j.jiac.2014.08.003.

649 Zhou, S., Xu, C., Wang, J., Gao, W., Akhverdiyeva, R., Shah, V., and Gross, R. 2004.

650 Supramolecular assemblies of a naturally derived sophorolipid. Langmuir 20(19): 7926651 7932. doi: 10.1021/la048590s.

652

653

654 
Fig. 1.

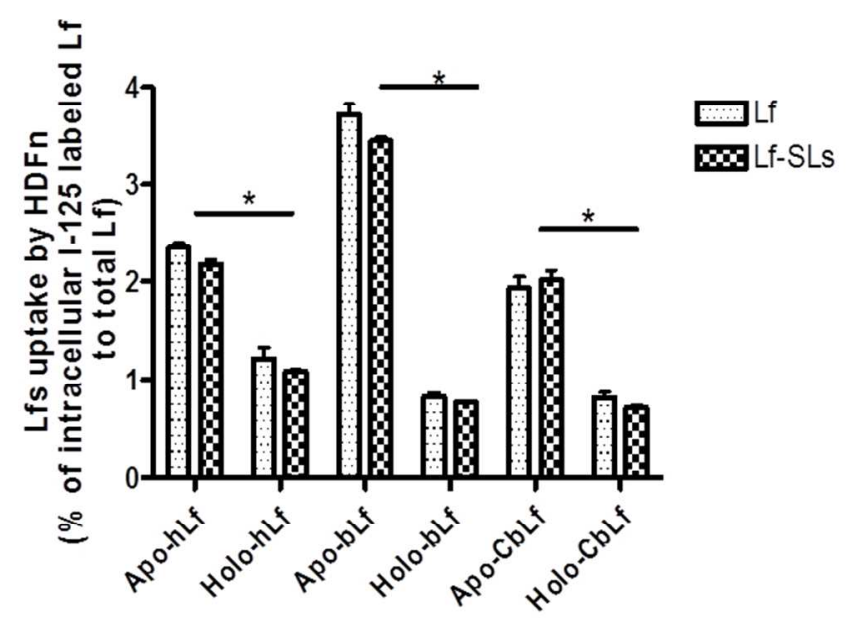

Fig.1. Uptake of Lfs by HDFn. After D10 HDFn were incubated in supplement-free medium containing 125Ilabeled Lf samples $\left(100 \mu \mathrm{g} / \mathrm{mL}\right.$ with or without SLs: $10 \mu \mathrm{g} / \mathrm{mL}$ ) for $30 \mathrm{~min}$ at $37^{\circ} \mathrm{C}$, cell-associated radioactivity was quantified in a gamma counter. Data are shown as means \pm SD for three independent experiments $(n=5)$. Differences between Lf and Lf-SLs-assembly groups were analyzed using one-way ANOVA. ${ }^{*} p<0.001$

$254 \times 190 \mathrm{~mm}(96 \times 96$ DPI) 


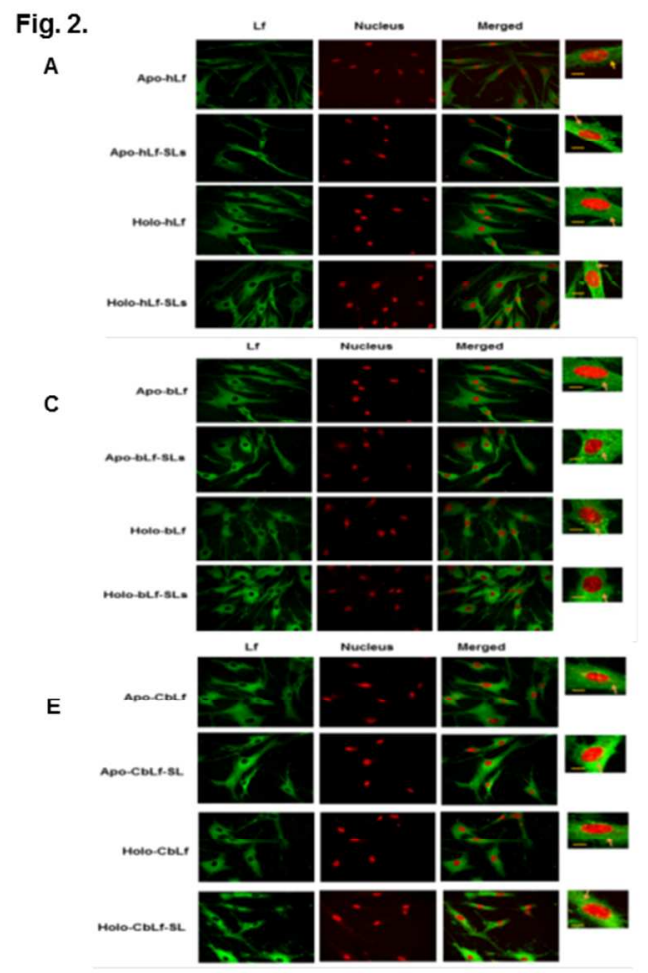

B

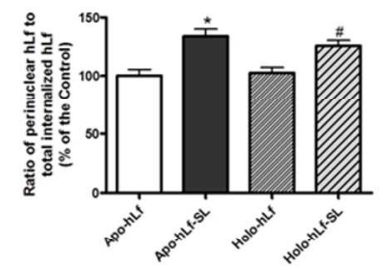

D

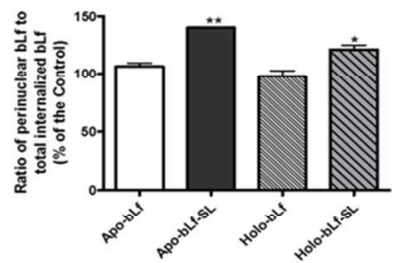

$\mathrm{F}$

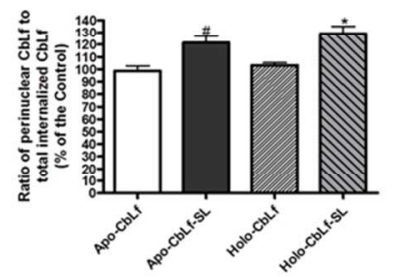

Fig. 2. Subcellular localization of internalized Lf in the presence or absence of SL. After cells were treated with Lfs $(100 \mu \mathrm{g} / \mathrm{mL})$ or Lf-SLs-assemblies in supplement free medium for 30 min at $37^{\circ} \mathrm{C}$, cells were stained with green fluorescence (Lf) and red fluorescence (nucleus). (A\&B): hLf, (C\&D): bLf, (E\&F): CbLf. Data are shown as means \pm SD for three independent experiments $(n=3)$. Differences between Lf and LfSLs-assembly groups were analyzed using one-way ANOVA. Scale bar: $10 \mu \mathrm{m}$. $* p<0.001, \# p<0.05$, $* * \mathrm{p}<0.01$. 
Fig. 3.

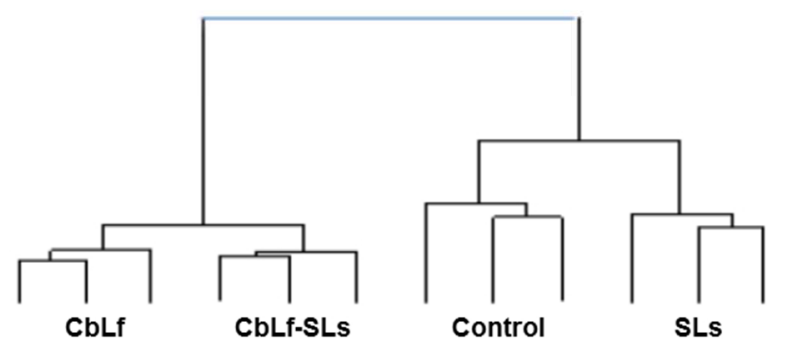

Fig. 3. Clustering of genes expressed in HDFs treated with Lf and SLs. Hierarchical clustering of 12 samples based on expression levels of 13716 probes detected in the microarray. Samples were clustered with a centroid linkage method on the horizontal axis, and the lengths of the branches were measure by a Euclidean algorithm. The vertical axis represents the degree of correlation between samples, and the lengths of the branches indicate similarities between samples or genes.

$254 \times 190 \mathrm{~mm}(96 \times 96 \mathrm{DPI})$ 


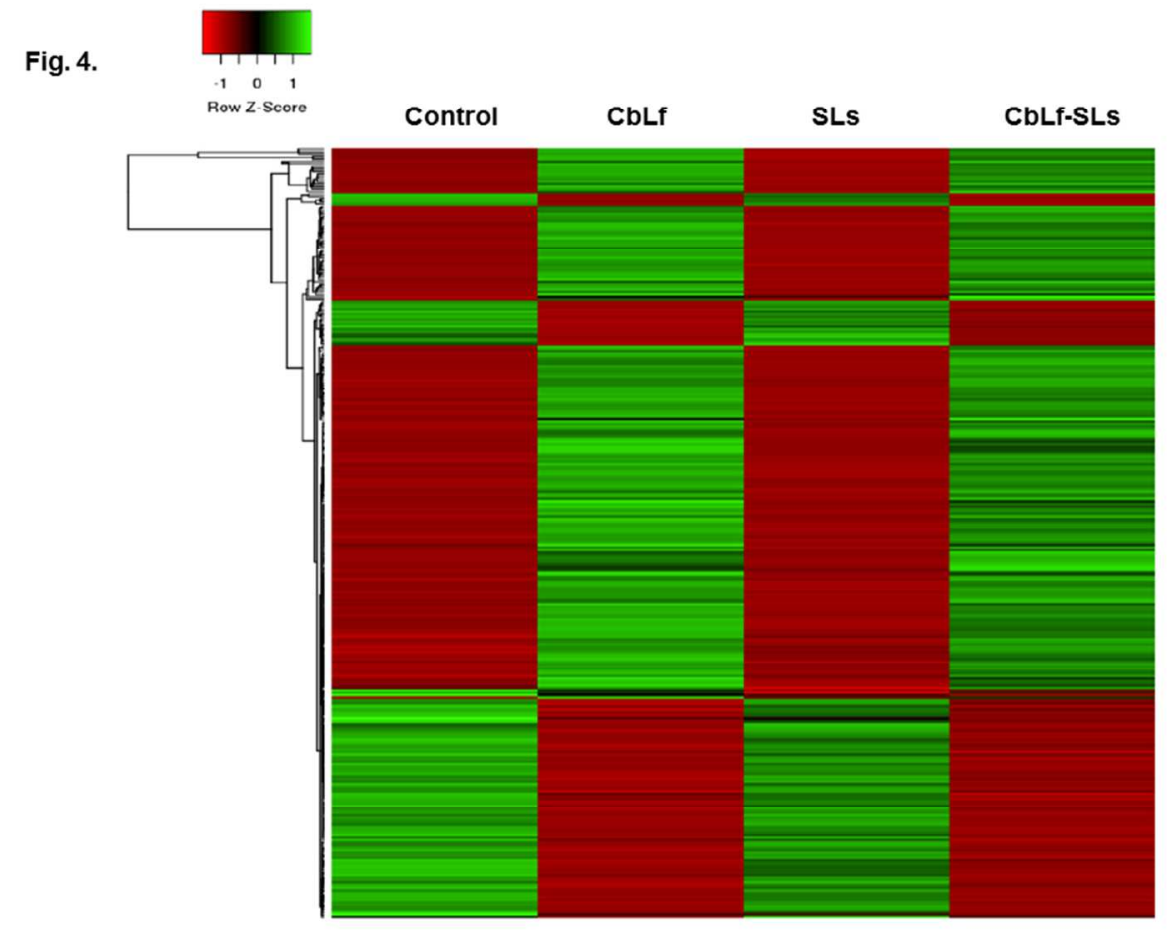

Fig. 4. Heatmap representation of the genes significantly regulated by CbLf, CbLf-SLs-assembly, and SLs. Probe sets for genes significantly regulated (adjusted $p$ value $<0.05$, fold change $\geq 3.0$ or $\leq 0.3$ ) were selected and reported in a heatmap. Each row represents a different probe set, and columns pertain to data collected from HDFn treated with CbLf, SLs, or CbLf-SLs-assembly.

$254 \times 190 \mathrm{~mm}(96 \times 96 \mathrm{DPI})$ 
Fig. 5 .

A

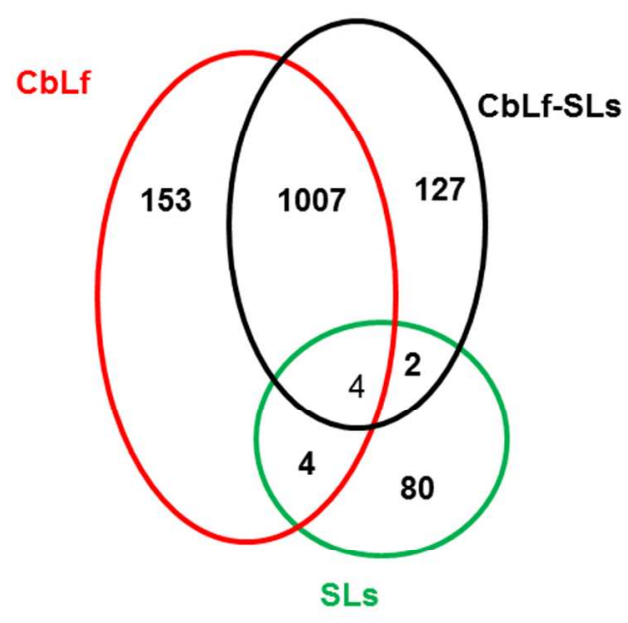

Up-regulated genes
B

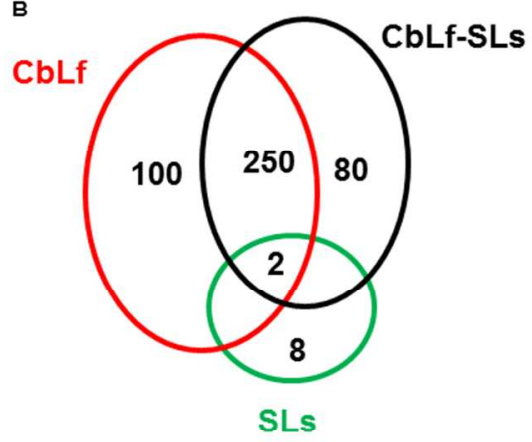

Down-regulated genes

Fig. 5. Global analysis of transcriptomic changes in the presence of CbLf, SLs, and CbLf-SLs-assembly indicated by Venn diagrams. Venn diagrams represent genes significantly regulated by CbLf, SLs, and CbLfSLs-assembly. Differentially expressed genes were selected following three criteria: fold change $>1.5$ for upregulation, fold-change $<0.5$ for down-regulation, and detection probability greater than 0.95 in all samples.

$254 \times 190 \mathrm{~mm}(96 \times 96 \mathrm{DPI})$ 
Fig. 6.
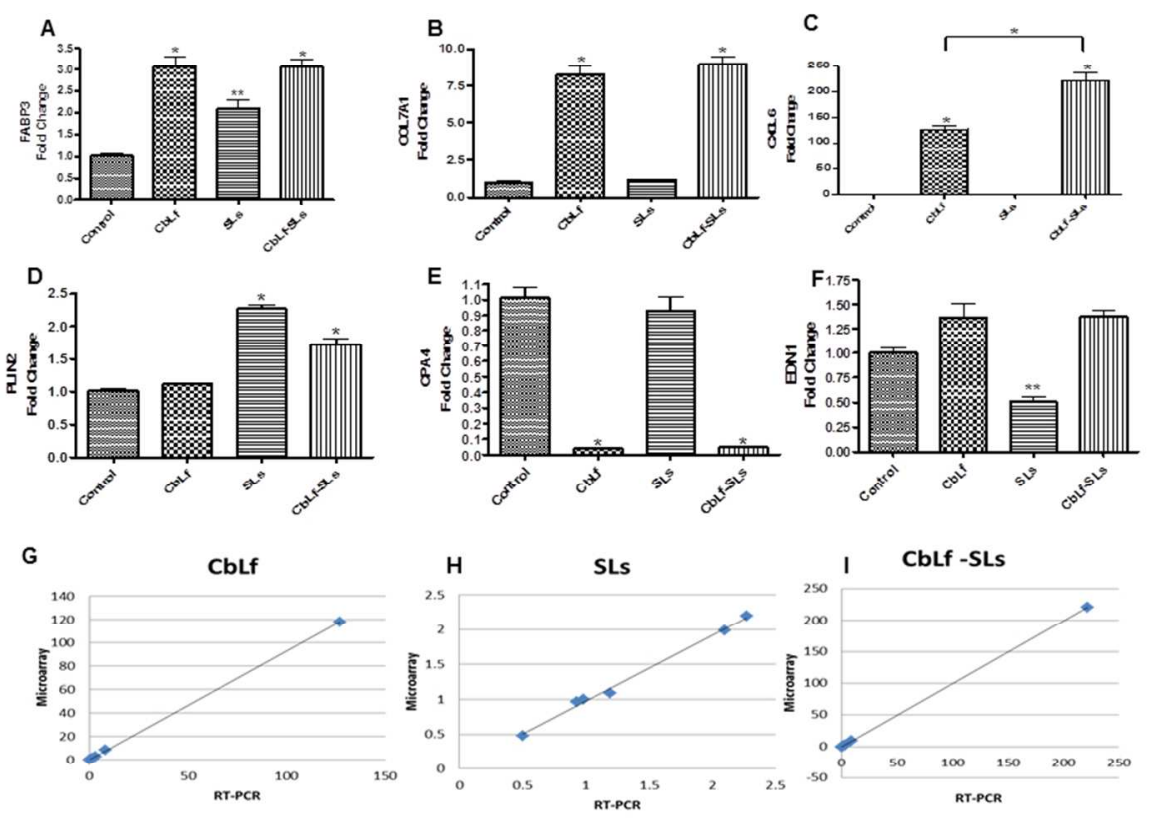

Fig. 6. Real-time PCR verification of microarray results. (A) FABP3 (B) COL7A1 (C) CXCL6 (D) PLIN2 (E) CPA4 (F) EDN1. (G) Associations between results from microarrays and RT-PCR were analyzed, and all were shown to have R2>0.95. Values are shown as mean fold-changes \pm SD for three independent experiments $(n=5)$, relative to control (non-treated control, set to 1$) . * p<0.001, * * p<0.01$

$$
254 \times 190 \mathrm{~mm}(96 \times 96 \mathrm{DPI})
$$


Fig. 7.

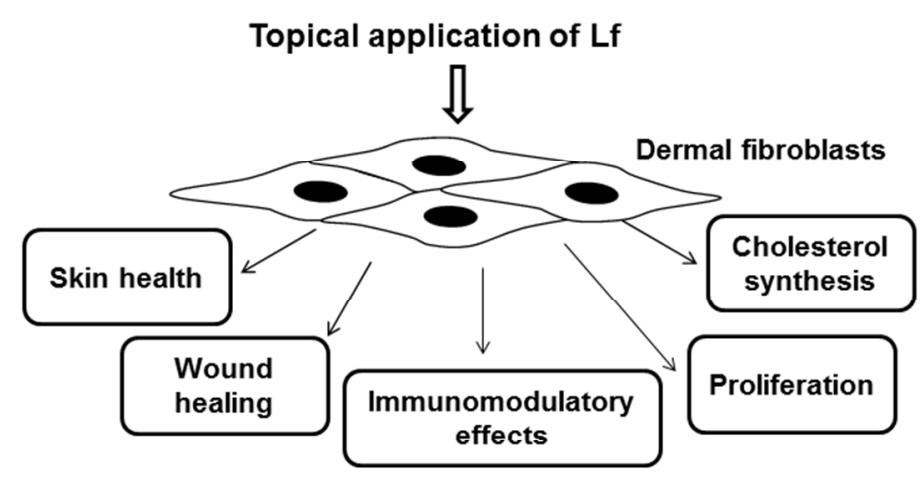

Fig. 7. Plausible physiological functions of topically applied CbLf and CbLf-SLs-assembly through activation of HDFn. According to the pathway analysis on microarray data, Lf is likely to activate HDFn to exert multiple functions such as anti-aging, wound healing, anti-microbial, cell proliferation, and cholesterol biosynthesis facilitating effects.

$254 \times 190 \mathrm{~mm}(96 \times 96 \mathrm{DPI})$ 
Table 1 Primers for Real-time PCR

\begin{tabular}{|l|l|}
\hline Primers & Sequence \\
\hline FABBP3 Forward & AGCCTAGCCCAGCATCACTA \\
\hline FABBP3 Reverse & AACCCACACCGAGTGACTTC \\
\hline COL7A1 Forward & CCCATGAGGCACCAGATACT \\
\hline COL7A1 Reverse & GCTGACTCCACCTTCGAGAC \\
\hline CXCL6 Forward & TGTTTACGCGTTACGCTGAG \\
\hline CXCL6 Reverse & ACCTTGCTTCCCGTTCTTCA \\
\hline PLIN2 Forward & GTCTCCTGGCTGCTCTTGTC \\
\hline PLIN2 Reverse & TCGGAAGTGGAGGTGAAATC \\
\hline CPA4 Forward & GTAGCTGTGCAGGTCGATGA \\
\hline CPA4 Reverse & TCCTCTGCTGGTTCCTGACT \\
\hline EDN1 Forward & ATGGAAGCCAGTGAAGATGG \\
\hline EDN1 Reverse & CAACGAATTTGGCTACAGCA \\
\hline GAPDH Forward & GGTGGTCCAGGGGTCTTACT \\
\hline GAPDH Reverse &
\end{tabular}

